\title{
Neonatal hypoglycemia after cesarean section
}

\author{
Hiroyuki Sumikura
}

Received: 20 January 2013/Accepted: 30 January 2013/Published online: 7 March 2013

(C) Japanese Society of Anesthesiologists 2013

Keywords Cesarean section - Reflex hypoglycemia . Preoperative fasting

This issue of the Journal of Anesthesia includes 2 reports of studies that investigated the effects of intravenous glucose infusions on neonates [1, 2]. Fukuda and colleagues [1] reported that they administered intravenous solutions (500 ml) containing 0,1 , or $5 \%$ glucose to women who underwent cesarean sections under epidural anesthesia. The solutions were given after the patients were in the operating room and before delivery. The investigators then measured the blood glucose levels of the mother, the umbilical cord blood, and the neonates. The women who did not receive glucose generally developed hypoglycemia, as did their neonates. The women who received $5 \%$ glucose generally developed hyperglycemia, and their neonates had reflex hypoglycemia. In the second report, Yatabe and colleagues [2] gave a continuous intravenous infusion containing 0 or $1 \%$ glucose to patients who underwent cesarean section under combined spinal and epidural anesthesia and measured blood glucose levels of the umbilical cord blood, and the neonates. The glucose levels of the umbilical cord blood were significantly different between the groups; however, the levels of the neonates were not significantly different $3 \mathrm{~h}$ after delivery.

Both these papers were accepted for publication because they convey important messages about current anesthesia

This comment refers to the article available at doi: 10.1007/s00540-012-1516-1, 10.1007/s00540-012-1512-5.

H. Sumikura $(\bowtie)$

National Center for Child Health and Development,

2-10-1 Okura, Setagayaku, Tokyo, Japan

e-mail: sumikura-h@ncchd.go.jp practice for cesarean sections in Japan, even though both studies are small, one is retrospective, and both have some issues with their methodology. In this editorial, I hope to explain the importance of these messages.

Excessive glucose load from a preoperative intravenous (IV) infusion may lead to neonatal hypoglycemia and has been described. Mendiola and colleagues [3] reported that neonates had a higher risk of having hypoglycemia when the maternal glucose level was more than $120 \mathrm{mg} / \mathrm{dl}$ or when the glucose load was $20 \mathrm{~g} / \mathrm{h}$ or more. Kenepp and colleagues [4] reported that a glucose load of $25 \mathrm{~g}$ or more resulted in maternal hyperglycemia and neonatal hypoglycemia when an intravenous solution with $5 \%$ glucose was used before cesarean section.

Neonatal hypoglycemia is assumed to be the end result of a hyperglycemic state in the mother. When glucose is given to the mother before delivery, it crosses the placenta to the fetus and, in excess, can result in fetal hyperglycemia and hyperinsulinemia, because the fetus must produce insulin to compensate for the excess glucose. After the cessation of the glucose supply at delivery, the neonate may develop reflex hypoglycemia, as neonatal insulin levels are still elevated. Because of this potential problem described in the classical literature [3, 4], non-glucosecontaining IV solutions have been generally used preoperatively for patients undergoing caesarian section. Unfortunately, it seems that few anesthesiologists are adequately aware of the risk of neonatal hypoglycemia caused by maternal glucose load.

In Fukuda's [1] study, a 25-g glucose load resulted in maternal and umbilical hyperglycemia and in hypoglycemia in the neonates. The women in this study received epidural anesthesia, and they were in the operating room a relatively long time before surgery or delivery. Therefore, the glucose levels from the glucose load did not rise as 
rapidly as they would have from a faster infusion. Recently, "co-loading" has become more common, i.e., patients are anesthetized solely by spinal anesthesia and rapidly given an intravenous infusion immediately after induction of anesthesia. In this situation, additional care should be taken because a more drastic increase in blood glucose levels could lead to a higher risk of reflex hypoglycemia in the neonate. When co-loading is used, glucosecontaining solutions (especially $5 \%$ preparations) might best be avoided.

Another issue raised by the 2 reports is the risk of maternal and neonatal hypoglycemia caused by excessive fasting before surgery. The risk of hypoglycemia associated with vaginal delivery is well recognized, and an appropriate glucose load is recommended for these mothers. However, not many studies have reported the risks of maternal and neonatal hypoglycemia associated with fasting before cesarean section [5]. Hypoglycemia of the mother and neonate associated with excessive fasting can be avoided in 2 ways. First, the mother can be given a glucose-containing solution to boost the neonate's blood glucose levels, as described by Fukuda et al. and Yatabe et al. [1, 2]. An appropriate maternal IV glucose load should maintain neonatal blood glucose levels without causing reflex hypoglycemia until the neonate develops the ability to produce or intake glucose. Both studies suggest that an intravenous solution with $1 \%$ glucose is suitable for this purpose. However, further studies are needed to investigate the optimal glucose load for mothers with gestational diabetes, for fetuses who are small or large for date, or for patients in whom co-loading is performed.

A second way to circumvent neonatal hypoglycemia is to avoid having women fast excessively before surgery. Traditionally, cesarean section had been thought to place mothers at high risk of aspiration pneumonia, and strict fasting was recommended. However, recent guidelines from the United States [6] and United Kingdom [7] have relaxed restrictions on oral intake before cesarean section.
The recommendation now is for oral intake to be the same as that for general surgery, unless a patient is at special risk. In Japan, it seems that many medical institutions still adhere to the stricter fasting practice. However, the more widespread practice of ERAS (Enhanced Recovery After Surgery) has brought with it a trend toward less excessive preoperative fasting, and I believe this offers us a good opportunity to review our practice in obstetric anesthesia.

To avoid neonatal hypoglycemia after a cesarean section, the mother needs to receive an appropriate amount of glucose. The anesthesiologist's duty is to give an appropriate glucose load before cesarean section, either orally or parenterally.

\section{References}

1. Fukuda I, Matsuda H, Sugahara S, Kazama T. The effect of intravenous glucose solutions on neonatal blood glucose levels after cesarean delivery. J Anesth. Epub ahead of print: 11 November, 2012.

2. Yatabe T, Tateiwa $H$, Ikenoue N, Kitamura S, Yamashita K, Yokoyama M. Influence of administration of $1 \%$ glucose solution on neonatal blood glucose concentration in cesarean section. Journal of Anesthesia. Epub ahead of print: November 7, 2012.

3. Mendiola J, Grylack LJ, Scanlon JW. Effects of intrapartum maternal glucose infusion on the normal fetus and newborn. Anesth Analg. 1982;61:32-5.

4. Kenepp NB, Kumar S, Shelley WC, Stanley CA, Gabbe SG, Gutsche BB. Fetal and neonatal hazards of maternal hydration with 5\% dextrose before caesarean section. Lancet. 1982;1:1150-2.

5. Marom R, Dollberg S, Mimouni FB, Berger I, Mordechayev N, Ochshorn $\mathrm{Y}$, et al. Neonatal blood glucose concentrations in caesarean and vaginally delivered term infants. Acta Paediatr. 2010;99:1474-7.

6. ASA. Practice guidelines for obstetric anesthesia: an updated report by the American Society of Anesthesiologists Task Force on Obstetric Anesthesia. Anesthesiology. 2007;106:843-63.

7. Gholitabar M, Ullman R, James D, Griffiths M. Caesarean section: summary of updated NICE guidance. BMJ. 2011;343:d7108. 